\title{
Research on Optimization of Regional Comprehensive Transportation Network Structure Based on Multi-objective Planning
}

\author{
Zhipeng Li ${ }^{1, \text { a }}$, Yuanhua Jia ${ }^{2, b}$ \\ ${ }^{1,2}$ School of Traffic and Transportation, Beijing Jiaotong University, Beijing 100044, China \\ a16120857@bjtu.edu.cn, ${ }^{b} 1141820498 @ q q . c o m$
}

Keywords: optimization, comprehensive transportation, transportation network structure, multi-objective planning, bi-level programming

Abstract: The paper, based on the practical problem of optimizing regional comprehensive transportation network structure, and referring to economics theory, uses multi-objective planning to propose an optimization model based on supply-demand equilibrium, minimizing generalized cost, and green sustainability. Due to more than two objective functions in the model, it is troublesome to convert the model into a single-objective programming problem. Therefore, the model is considered to be converted into a bi-level programming problem. Based on the genetic algorithm, the specific algorithm for solving the model is given and conducted a simulation calculation. The results shows that the proposed method in this paper is verified to solve practical problem.

\section{Introduction}

With the transformation of our nation's economic development mode and the adjustment of industrial structure, the relationship between transportation and the economic and social have gradually transferred into the construction adaptation from the total size adaptation. With the acceleration of the pace of transformation, the structural contradiction of the regional comprehensive transportation network has intensified, which has seriously affected the efficiency of transportation resources' allocation. Some scholars have noticed similar problems, for example, Basso, Leonardo J and Jara-Diaz, Sergio R analyzed the index of the transport structure from the economic point of view. It is considered that the density of the economy and the scope of the economy are the two basic indicators of transport structure [1]; Baiding Hu, Michael McAleer considered that the transportation industry should be incorporated into the industrial structure for research [2].

In recent years, around the optimization of the structure of the transportation system, some achievements have been made by the academic community based on relevant mathematical models and algorithms. About the specific issues in the structural aspects of transportation systems, Mark W. Homer, Morton E. O'Kelly studied on how to realize the optimization of space configuration of 
service systems for container ports [3]; Illi Racunica, Laura Wynter, etc. did in-depth research on how to realize the optimization of transportation system structure and the effective allocation of transportation resources from the aspects of economies of scale, etc. [4]; In terms of model solution, in order to solve the problem about imbalance of Japanese specialized container hub structures, Akio Imai and Etsuko Nishimura, etc. revised the existing model of the berth structure of the port, and used the genetic algorithm technology to solve nonlinear problems [5].

The paper is based on the practical problem of optimizing regional comprehensive transportation network structure, and, referring to economics theory, uses multi-objective planning to propose an optimization model. Then, the model is considered to be converted into a bi-level programming problem. Based on the genetic algorithm, the specific algorithm for solving the model is given and conducted a simulation calculation. The results show that the proposed method in this paper is verified to solve practical problem.

\section{Problem Description}

In terms of Economics, the optimized configuration of the regional comprehensive transportation network structure must seek to the equilibrium of supply and demand, namely, to meet the needs of users choosing different modes of transportation to travel. It is also necessary to meet the objective of optimizing the system's efficiency, that is, to reduce the generalized expenses for users to travel in different modes of transportation as much as possible. At the same time, from the standpoint of sustainable development of the system, it is necessary to guide the growth of environmentally friendly, resource-saving modes of transportation and suppress excessive development of the high-consumption, high-pollution transportation. The paper attempts to achieve the goal that meets a stable demand for passenger and freight transportation, and at the same time plays a guiding role for the travel of passenger and freight, in a more sustainable way under limited resource input. Sustainable development strategy. Therefore, there is a need to establish a multi-objective optimization programming model of regional comprehensive transportation network structure, which is based on supply-demand equilibrium, minimizing generalized cost, and green sustainability.

\section{Mathematical Model}

\subsection{Section Headings}

For decision makers, the purpose of adjusting the structural configuration of the regional comprehensive transportation network is to invest limited funds in the mode of transportation with insufficient supply capacity so that the ratio of supply and demand for various modes of transport tends to be stable, that is, approaches l, to build a harmonious and stable comprehensive transportation system. The existing modes of transportation often includes both passenger and cargo transportation. For example, there are both passenger and freight transport on the road. Due to the differences in the technical and economic characteristics of various modes of transport and the differences between passengers and cargo itself, passenger and freight transport are often different. It is necessary to discuss separately. This requires that the investment in construction of transport infrastructure not only meet the passenger supply-demand equilibrium, but also meet the freight supply-demand equilibrium.

So the goal of function is to make the passenger transport capacity of various modes of transport infinitely close to the demand of passenger travel and the cargo transport capacity of various transport modes infinitely close to the freight transport demand, that is, the objective function is: 


$$
\min Z=w_{1} Z_{1}+w_{2} Z_{2}
$$

Where $w_{1}$ and $w_{2}$ are parameters as the priority of $Z_{1}$ and $Z_{2}$, and

$$
Z_{1}=\sum_{i=1}^{n}\left|\frac{\left(I r_{i} / b_{i}+Z_{i}\right) c_{i 1}}{X_{i 1}}-1\right|
$$

Where $n$ is the number of types of transportation, $I$ is total investment in transport infrastructure within the regional comprehensive transport system, $r_{i}$ is the proportion of investment in the $i$-th mode of transport, $b_{i}$ is the average unit line construction cost for the $i$-th mode of transport, $Z_{i}$ is the original traffic mileage of the $i$-th type of transportation before investment, $c_{i 1}$ is unit passenger capacity of the $i$-th mode of transport, and $X_{i 1}$ is passenger demand for the $i$-th mode of transport.

$$
Z_{2}=\sum_{i=1}^{n}\left|\frac{\left(I r_{i} / b_{i}+Z_{i}\right) c_{i 2}}{X_{i 2}}-1\right|
$$

Where $c_{i 2}$ is unit freight capacity of the $i$-th mode of transport, $X_{i 2}$ is cargo demand for the $i$-th mode of transport.

\subsection{Objective Function Based on Minimizing Generalized Cost}

The generalized cost of transportation is an important factor about transportation efficiency in the comprehensive evaluation system. It is a comprehensive reflection of transportation costs, transportation time, transit costs, transfer time and other factors. It shows the final currency value of each factor through a certain conversion relationship. Broadly used costs were initially used in road traffic network planning and are comprehensive evaluations of all the attributes of road service levels. The service level attributes generally include such factors as transportation distance, travel time, flow-speed relationship, safety and comfort, and other expenses (such as fuel costs, tolls, etc.).

So the goal of function is to reduce generalized cost for passenger travel and freight as much as possible, that is, the objective function is:

$$
\min Q=\varphi_{1} Q_{1}+\varphi_{2} Q_{2}
$$

Where $\varphi_{1}$ and $\varphi_{2}$ are parameters as the priority of $Q_{1}$ and $Q_{2}$, and

$$
Q_{1}=\sum_{i=1}^{n} C_{i}=\sum_{i=1}^{n}\left(E_{i} \cdot\left(Z_{i}+I r_{i} / b_{i}\right)+F_{i}+N_{i}+M_{i}+S_{i}\right)
$$

And

$$
\begin{aligned}
& F_{i}=\left(T_{i 1}+T_{i 1}{ }^{\prime}\right) V_{1}(T) \\
& N_{i}=\left(W_{i}+Y_{i}\right) V_{1}(T)
\end{aligned}
$$

Where $C_{\mathrm{i}}$ is the generalized passenger travel cost of the $i$-th mode of transport, $E_{i}$ is unit passenger travel price for the $i$-th mode of transport, $F_{i}$ is rapidity costs of the $i$-th mode of transportation, $N_{i}$ is convenience costs of the $i$-th mode of transport, $M_{i}$ is comfort costs for the $i$-th mode of transport, $S_{i}$ is security costs for the $i$-th mode of transport, $T_{i 1}$ is travel time for the $i$-th mode of transport, $T_{i 1}$ ' is the time required for the passengers to connect with the $i$-th transportation mode, $V_{1}(T)$ is time value of a passenger, $W_{i}$ is ticket purchase time for the $i$-th transportation method, and $Y_{i}$ is The average 
turnaround time for the $i$-th mode of transport.

$$
Q_{2}=\sum_{i=1}^{n} C_{i 2}=\sum_{i=1}^{n}\left(E_{i 2} \cdot\left(Z_{i}+I r_{i} / b_{i}\right)+S_{i 2}+F_{i 2}+L_{i 2}\right)
$$

And

$$
F_{i 2}=\left(T_{i 2}+T_{i 2}{ }^{\prime}\right) V_{2}(T)
$$

Where $C_{i 2}$ is generalized freight cost for the $i$-th mode of transport, $E_{i 2}$ is unit freight price for the $i$-th mode of transport, $L_{i 2}$ is cost for goods' stevedoring of the $i$-th transportation method at the pick-up point, $F_{i 2}$ is time cost of the $i$-th mode of transport (transportation time, stevedoring time), $S_{i 2}$ is the security cost of the $i$-th transportation method, $T_{i 2}$ is average transit time of the $i$-th mode of transport, $T_{i 2}$ ' is time for goods' stevedoring of the $i$-th transportation method at the pick-up point, and $V_{2}(T)$ is time value of cargo transportation.

\subsection{Objective Function Based on Green Sustainability}

Due to the differences in the means of transportation, its energy consumption and the pollution to the environment are different when transporting the same weight goods. From the perspective of the entire transportation system, we should encourage the development of transportation modes with low energy consumption and less environmental pollution, and actively build the corresponding infrastructure which can undertake more transportation. This paper selects energy consumption objective function to reflect the sustainable development capacity of the comprehensive transport system. The function means that under a certain proportion of investment, the minimum energy is consumed when the new-build infrastructure complete the same transportation, that is, the objective function is:

$$
\min G=\sum_{i=1}^{n} I r_{i} / b_{i}\left(c_{i 1}+c_{i 2}\right) d_{i}
$$

Where $d_{i}$ is energy consumption of unit capacity of the $i$-th mode of transport.

Do the normalization of $d_{i}$, and the calculation formula is:

$$
u_{i}=d_{i} / \sum_{i=1}^{n} d_{i}
$$

Then do the normalization of the objective function, the calculation formula is:

$$
\min G^{\prime}=\frac{\sum_{i=1}^{n} I r_{i} / b_{i}\left(c_{i 1}+c_{i 2}\right) u_{i}}{\sum_{i=1}^{n} I r_{i} / b_{i}\left(c_{i 1}+c_{i 2}\right)}
$$

\subsection{Multi-objective Optimization Programming Model}

All of the above objective functions have their constraints, and only the solutions that satisfy the constraints are the feasible solutions and the optimal solutions. There are two kinds of constraints.

First, there is system constraints, which refer that the sum of the investment ratios of various transport modes is l, as consideration of all transport modes within the transportation system, that is: 


$$
\sum_{i=1}^{n} r_{i}=1
$$

Second, there is a scope constraint, which refers that the investment ratio of various modes of transport should be a number between 0 and 1, that is:

$$
\begin{aligned}
0<r_{i} & <1 \quad i=0,1,2, \ldots, n \\
0 & <I<\mathrm{N}
\end{aligned}
$$

Where $N$ is number of the largest investment in the region.

Therefore, the form of multi-objective optimization programming model of regional comprehensive transportation network structure is as follows:

$$
\begin{gathered}
\min Z=w_{1} \sum_{i=1}^{n}\left|\frac{\left(I r_{i} / b_{i}+Z_{i}\right) c_{i 1}}{X_{i 1}}-1\right|+w_{2} \sum_{i=1}^{n}\left|\frac{\left(I r_{i} / b_{i}+Z_{i}\right) c_{i 2}}{X_{i 2}}-1\right| \\
\min Q=\varphi_{1} \sum_{i=1}^{n}\left(E_{i} \cdot\left(Z_{i}+I r_{i} / b_{i}\right)+\left(T_{i 1}+T_{i 1}^{\prime}+W_{i}+Y\right) V_{1}(T)+M_{i}+S_{i}\right)+\varphi_{2} \sum_{i=1}^{n}\left(E_{i 2} \cdot\left(Z_{i}+I r_{i} / b_{i}\right)+S_{i 2}+\left(T_{i 2}+T_{i 2}{ }^{\prime}\right) V_{2}(T)+L_{i 2}\right)(17) \\
\min G^{\prime}=\frac{\sum_{i=1}^{n} I r_{i} / b_{i}\left(c_{i 1}+c_{i 2}\right) u_{i}}{\sum_{i=1}^{n} I r_{i} / b_{i}\left(c_{i 1}+c_{i 2}\right)}
\end{gathered}
$$

The constraints is:

$$
\text { s.t. }\left\{\begin{array}{l}
\sum_{i=1}^{n} r_{i}=1 \\
0<r_{i}<l \quad i=0,1,2, \ldots, n \\
0<I<N
\end{array}\right.
$$

\section{Solution for the Model and Algorithm}

\subsection{Transformation of the Model}

Due to more than two objective functions in the model, it is troublesome to convert the model into a single-objective programming problem. Therefore, the model is considered to be converted into a bi-level programming problem.

The model is divided into two layers: the upper layer is an objective function based on supply-demand equilibrium and minimizing generalized cost, and the lower layer is an objective function based on green sustainability, as follows.

The upper layer:

$$
\min (Z, Q)
$$




$$
\text { s.t. }\left\{\begin{array}{l}
\sum_{i=1}^{n} r_{i}=1 \\
0<I<N
\end{array}\right.
$$

The lower layer:

$$
\begin{gathered}
\min G^{\prime} \\
\text { s.t. }\left\{\begin{array}{l}
0<r_{i}<1 \quad i=0,1,2, \ldots, n \\
0<I<N
\end{array}\right.
\end{gathered}
$$

\subsection{Design of Algorithm}

From the above, the basic form of the model is:

$$
\left\{\begin{array}{l}
\min _{x \in X}\left(A_{1}(\mathrm{x}, \mathrm{y}), A_{2}(\mathrm{x}, \mathrm{y}), \cdots, A_{m}(\mathrm{x}, \mathrm{y})\right) \\
\text { S.T. } G(\mathrm{x}, \mathrm{y}) \leq 0 \\
\min _{y \in Y} a(\mathrm{x}, \mathrm{y}) \\
\text { S.T. } \mathrm{g}(\mathrm{x}, \mathrm{y}) \leq 0
\end{array}\right.
$$

The following assumptions are made for the established model:

1) $S$ is non-empty, guaranteeing the existence of solutions;

2) It is assumed that the lower optimal solution is unique for each upper variable. If the lower optimal solution is not unique, it is necessary to select a suitable lower optimal solution according to the preference of the upper decision maker for ease of calculation.

In view of the advantages of genetic algorithm in solving the problems based on complex bi-level programming, this paper designs a genetic algorithm program for solving the model [6].

Step 0: randomly select $M$ points in $X, x_{i}=\left(x_{i}^{1}, x_{i}^{2}, \cdots, x_{i}^{\alpha}\right), i=1,2, \cdots m$ and use the genetic algorithm to calculate the corresponding optimal solution of the lower layer, $y_{i}=\left(y_{i}^{1}, y_{i}^{2}, \cdots, y_{i}^{\beta}\right), i=1,2, \cdots m$, to obtain the interpolation function $\varphi(x)=\left(\varphi^{1}(x), \varphi^{2}(x), \cdots, \varphi^{\beta}(x)\right)$;

Step 1: Extract $M_{1}$ points from $X$, and bring these points into the interpolation function $\varphi(\mathrm{x})$ to get the initial population with population size $N_{1}$ [7]:

$$
\operatorname{group}(0)=\left(\begin{array}{cccccccc}
x_{1}^{1} & x_{1}^{2} & \cdots & x_{1}^{\alpha} & \varphi^{1}\left(\mathrm{x}_{1}\right) & \varphi^{2}\left(\mathrm{x}_{1}\right) & \cdots & \varphi^{\beta}\left(\mathrm{x}_{1}\right) \\
x_{2}^{1} & x_{2}^{2} & \cdots & x_{2}^{\alpha} & \varphi^{1}\left(\mathrm{x}_{2}\right) & \varphi^{2}\left(\mathrm{x}_{2}\right) & \cdots & \varphi^{\beta}\left(\mathrm{x}_{2}\right) \\
\vdots & \vdots & \cdots & \vdots & \vdots & \vdots & \cdots & \vdots \\
x_{m}^{1} & x_{m}^{2} & \cdots & x_{m}^{\alpha} & \varphi^{1}\left(\mathrm{x}_{m}\right) & \varphi^{2}\left(\mathrm{x}_{m}\right) & \cdots & \varphi^{\beta}\left(\mathrm{x}_{m}\right)
\end{array}\right)
$$

And let $r=0$;

Step 2: From the group $(r)$, select the parents that participated in the cross. The probability of crossing is $P_{c}$, and the descendant set is $G_{1}$. Under the specific hybridization process, let $X_{1}$ and $X_{2}$ be parent individuals, where $\lambda \in(0,1)$;

Step 3: From the group $(r)$, select the parents that participated in the mutation, the mutation 
probability is $P_{s}$, and the offspring set is $G_{2}$. The mutation process is as follows: If $x=\left(x^{1}, x^{2}, \cdots, x^{\alpha}\right)$ are selected to participate in mutation, at first, give the interval where each component is located, $x^{i} \in\left[\sigma^{i}, \mu^{i}\right], i=1,2, \cdots, \alpha$. Randomly generate a random number according to the uniform distribution, $\theta_{i} \in[0,1], i=1,2, \cdots, \alpha$, then

$$
x^{\mathrm{r}}=\left\{\begin{array}{lr}
\text { Randomly generate } \overline{x^{\mathrm{r}}} \in\left[\sigma^{\mathrm{i}},\right. & \left.\mu^{\mathrm{i}}\right] \text { if } \theta_{\mathrm{r}} \leq P_{S} \\
X^{r} & \text { if } \theta_{\mathrm{r}} \geq P_{S}
\end{array}\right.
$$

The mutated offspring set are $\bar{x}=\left(\overline{x^{1}}, \overline{x^{2}}, \cdots, \overline{x^{\alpha}}\right)$ [8].

Step 4: Carry out a fast non-dominant sort for each point in $H=\left\{\operatorname{group}(r) \cup G_{1} \cup G_{2}\right\}$ and determine each individual's rank order value. Calculate the crowding degree for individuals with the same ordinal value. Then, each individual gets two attributes of $i \operatorname{Irank}\left(i_{\text {rank }}\right)$ and crowding degree $\left(i_{d}\right)$, which can deterne the individual's superiority and inferiority relation according to these two attributes, when satisfy $i_{\text {rank }}<j_{\text {rank }}$, or $i_{\text {rank }}=j_{\text {rank }}$, and $i_{d}>j_{d}$, say $i \prec j$. With the method above, $M$ individuals are selected from $H$ as the next-generation population group $(r+1)$ [9].

Step 5: Select the individuals with $i_{\text {rank }}=1$ (assuming there are $m$ ) from the descendant set, correct the lower optimal solutions of the $m$ individuals, and update the interpolation function with these points as the new interpolation points. In order to reduce the amount of calculations in the correction process, a method for evaluating multiple criteria can be designed: First, for each point $x$, generate $t$ points according to a Gaussian distribution. Then, obtain a population with $t \bullet m$ as the population size. In the process of evolution and selection, $m$ fitness functions are generated by substituting $m$ upper-level decision variables into the lower objective function. [10].

Step 6: If the algorithm reaches the maximum algebra, or if the results of the 20th generation are not improved, the algorithm stops; otherwise, let $r=r+1$, and go to step 2.

\section{Simulation}

It is assumed that within a certain area $H B$, there are four modes of transportation, such as, railways, highways, inland river shipping, and aviation, and the technology level of transportation within a certain period of time remains unchanged, and the energy consumption level of each mode of transportation remains unchanged. Referring to reports on the government's work, statistical yearbooks, transportation-related planning and transportation-related data in the region, the model parameters can be determined. Some of the model parameters in the model are shown in Table 1.

What's more, for ease of calculation, consider the objective functions $Z_{1}$ and $Z_{2}$ as equally important, that is, $w_{1}=w_{2}=0.5$, and similarly, let $\varphi_{1}=\varphi_{2}=0.5$.

In the multi-objective genetic algorithm, the population size is 100 , the largest generation population size is 2000, the crossover rate is 0.1 , and the mutation rate is 0.3 . According to the previous solution steps, the Parato frontiers of the multi-objective programming model can be obtained by programming calculation with MATLAB software, as shown in Figure 1. Finally, the results of optimization in this area are determined as shown in Table 2. 
Table 1 Model Parameters Value

\begin{tabular}{c|c|c|c|c}
\hline Parameter & Railway & Highway & $\begin{array}{c}\text { Inland river } \\
\text { shipping }\end{array}$ & Aviation \\
\hline$i$ & 1 & 2 & 3 & 4 \\
\hline$X_{i 2}$, million t & 5231 & 4103 & 5012 & 900 \\
\hline$E_{i}, Y / \mathrm{km}$ & 0.15 & 0.2 & 0.08 & 1.05 \\
\hline$T_{i 1}, \mathrm{~h}$ & 1.52 & 0.84 & 1.33 & 2.11 \\
\hline$Y_{i}, \mathrm{~h}$ & 0.51 & 0.17 & 0.83 & 1.01 \\
\hline$E_{i 2}, Y / \mathrm{km}$ & 0.3 & 0.5 & 0.1 & 0.8 \\
\hline$V_{1}(T), Y / \mathrm{h}$ & \multicolumn{5}{|l}{18.5} \\
\hline $\begin{array}{c}d_{i}, \mathrm{~kg} \text { of standard coal } \\
/(\text { thousand people- } \\
\text { km) }\end{array}$ & 1.6 & 20 & 8 & 500 \\
\hline$u_{i}$ & 0.111 & 0.555 & 0.056 & 0.278 \\
\hline$N$,million $¥$ & \multicolumn{5}{|l}{430} \\
\hline
\end{tabular}
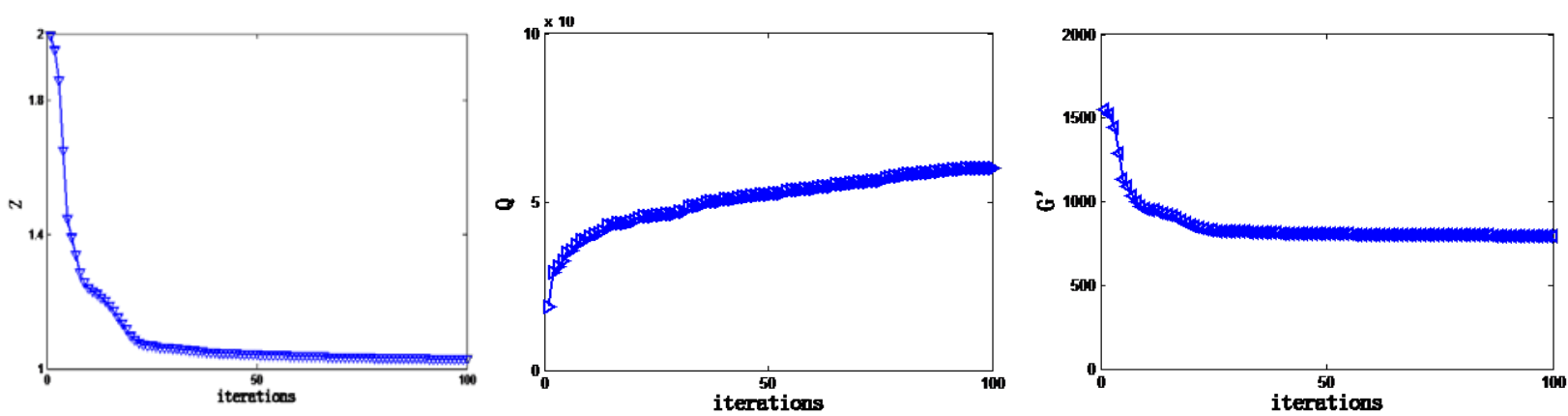

Fig. 1 the Parato frontiers of the multi-objective programming model

Table 2 the Results of Optimization

\begin{tabular}{c|c|c|c|c}
\hline Optimum solution & Railway & Highway & $\begin{array}{c}\text { Inland river } \\
\text { shipping }\end{array}$ & Aviation \\
\hline Investment ratio & $17.88 \%$ & $70.33 \%$ & $3.61 \%$ & $8.18 \%$ \\
\hline $\begin{array}{c}\text { Investment amount,million } \\
\text { yuan }\end{array}$ & 70.4472 & 277.1002 & 14.2234 & 32.2292 \\
\hline Total investment,million yuan & \multicolumn{4}{|c}{394} \\
\hline
\end{tabular}

\section{Conclusions}

The optimization of regional comprehensive transportation structure is a dynamic optimization process. The multi-objective programming model is used to abstract the problem of regional comprehensive transportation structure optimization into a mathematical problem, which is of certain scientificalness. This paper selects a multi-objective genetic algorithm to solve the model, and the example application of the algorithm can get a more uniform distribution of pareto frontiers, which converge faster and have better stability. So it is suitable for solving bi-level multi-objective programming problems. 


\section{Acknowledgments}

The research was supported in part by my tutor Yuanhua Jia .His valuable advice helps me to improve my paper.

\section{References}

[1] Basso, Leonardo J, Jara-Diaz, Seigio R, Are returns to scale with variable network size adequate for transport industry structure analysis [J]. Transportation Science 2006.

[2] Balding Hu, Michael McAleer. Input-output structure and growth in China [J]. Mathematics and Computers in Simualtion 64(2004)193-202.

[3] Mark W.Homer, Morton E.O'Kelly. Embedding economies of scale concepts for hub network design. Journal of Transport Geography, V9, 2001:255-265.

[4] Illia Racunica, Laura Wynter. Optimal location of intermodal freight hubs [J]. Transportation Research Part B, V39, 2005:453-477.

[5] Akio Imai, Etsuko Nishimura, Stratos Papa dimituiou. Berth allocation in a container port: using a continuous location space approach [J]. Transportation Research Part B, V39, 2005:199-221.

[6] Xihui Yin. Research on Theory and Method of Passenger Transportation Structure Optimization Based on Evolution Mechanism [D]. Beijing Jiaotong University, 2015.

[7] Zitzler E, Thiele L. Multi objective evolutionary algorithms: a comparative case study and the strength Pareto approach [J]. IEEE Transactions on Evolutionary Computation, 1999, 3(4): 257-271.

[8] Zitzler E, Laumanns M, Thiele L. SPEA2: Improving the strength pareto evolutionary algorithm for multi-objective optimization [C]//Proceedings of the Evolutionary Methods for Design , Optimization and Control, Barcelona, Spain, 2002: 19-26.

[9] Corne D W, Knowles J D, Oates M J. The pareto envelope-based selection algorithm for multi-objective optimization[C]//Proceedings of the 6th International Conference on Parallel Problem Solving from Nature, Berlin, 2000: 839-848.

[10] Deb K, Agrawal S, Pratap A, et al. A fast and elitist multi-objective genetic algorithm: NSGA-II[J]. IEEE Transactions on Evolutionary Computation, 2002, 6(2): 182-197. 